\title{
La biodiversidad en tiempos de crisis. Algunas propuestas para Europa
}

\author{
Eladio Fernández Galiano ${ }^{1}$
}

Sumario: I. NUEVOS RETOS PARA LA BIODIVERSIDAD. II. LOS SERVICIOS DE LOS ECOSISTEMAS, NUEVO LEITMOTIV DE LA BIODIVERSIDAD. III. RETOS PARA EL FUTURO EN EUROPA. HISTORIA DE LOGROS Y FRACASOS. IV. LO QUE QUEDA POR HACER: ALGUNAS PRIORIDADES PARA LA BIODIVERSIDAD EUROPEA. 1. El cambio climático. 2. La integración de la biodiversidad en políticas sectoriales. 3. Cambiar el modelo de zonas protegidas. 4. No olvidar las especies. 5. Combatir las especies exóticas invasoras. 6. Un pacto mundial por los océanos. 7. Controlar el ladrillo, la construcción ubicua de infraestructuras, y otros males: hacia unos nuevos hábitos de consumo sostenibles.

No corren buenos tiempos para la biodiversidad en Europa. Diecisiete años después de la adopción de los dos textos internacionales decisivos (el Convenio Sobre la Diversidad Biológica firmado en la Cumbre de la Tierra de Río de Janeiro y la Directiva Hábitats de la Unión Europea, ambos de 1992) nos encontramos ante un panorama desesperanzador. Se siguen perdiendo especies y hábitats naturales a un ritmo creciente, los bosques siguen con una silvicultura tradicional poco respetuosa de las especies autóctonas o se trasforman en áreas para biocarburantes u otros cultivos, nuevas urbanizaciones ocupan espacios naturales, los paisajes rurales siguen su constante proceso de artificialización y banalización, las pesquerías se derrumban por sobrepesca, las costas desaparecen bajo una muralla de cemento, los suelos pierden fertilidad y sólo las zonas protegidas permiten el refugio precario de una biodiversidad cada vez más rala, amenazada por una crisis ambiental y climática que no parecemos saber manejar.

Los compromisos del 92 se han quedado en poca cosa. En Río se nos prometía un plan de acción mundial para promover el desarrollo sostenible, una ordenación de la explotación de los bosques del mundo que asegurara su conservación, una mejora

1 Jefe de la Unidad de Diversidad Biológica del Consejo de Europa NB. Las opiniones expresadas en este artículo son exclusivamente personales y no reflejan las del Consejo de Europa. 
espectacular de la diversidad biológica, protegida por un convenio mundial, la limitación del calentamiento climático, el control de la desertificación. Tres convenios internacionales iban a asegurarnos un futuro verde y sostenible. Todo ello unido a un cambio de las modalidades de producción y de consumo, el fin de la pobreza, el uso racional de los recursos naturales, el fin de la sobreexplotación de los océanos y una agricultura respetuosa del medio ambiente y atenta a la conservación de los suelos. Todo ello era el famoso Programa 21 que debía prepararnos para este siglo nuevo que se anunciaba ecológico, próspero y verde.

El siglo despertó, sin embargo, explosivo y guerrero un 11 de Septiembre a orillas del Hudson y el entusiasmo hacia la sostenibilidad se fue progresivamente diluyendo en preocupaciones de seguridad y ansias de crecimiento económico. En Europa la creación de una moneda única, que empezaría a circular en el 2002, y la incorporación de doce nuevos miembros, ayudaron un poco más a hacer caer las barreras y nuestra economía se globalizó. Vinieron años de prosperidad económica, pero ¿que fue de los sueños de sostenibilidad? En la medida en que esta podía condicionar el crecimiento, fue aparcada. Se puede afirmar sin exagerar que los gobiernos europeos no han aprovechado los años de bonanza para cambiar el modelo hacia una mayor sostenibilidad. Más bien al contrario. Con rara unanimidad los gobiernos europeos de uno u otro signo han apostado con entusiasmo -sobre todo en nuestro país- por promover el consumo de bienes, la urbanización de las costas más allá de todo límite racional (racional no sólo ya en lo ambiental, sino en lo puramente económico) como si el planeta fuera ilimitado, como si las nuevas casas no necesitaran agua, energía, espacio, elementos todos ellos que se van haciendo raros y preciosos en nuestro continente.

Ahora con la crisis económica, se redescubren las virtudes del ahorro energético, de limitar nuestros desplazamientos, se vuelve la vista a valores que creíamos olvidados. Lo ambiental quiere volver a ponerse de moda. Hasta la biodiversidad empieza a presentarse en público como un posible recurso y no sólo como una carga para el erario público. Por desgracia no parecen quedar muchos dineros en la caja común para asegurar su conservación, por muchos beneficios sociales y muchos "servicios ecosistémicos" que genere.

\section{NUEVOS RETOS PARA LA BIODIVERSIDAD}

En marzo del 2009, el gobierno alemán, que en la actualidad preside el Convenio de la Diversidad Biológica de las Naciones Unidas, invitó a cincuenta personas de todo el mundo a una reunión en Bonn para que dieran ideas sobre lo que deberían ser los nuevos objetivos del Convenio a partir del 2010. Ese año, como se sabe, es un año crítico para la conservación de la naturaleza y de la diversidad biológica. El 2010 ha sido declarado por la Asamblea General de Naciones Unidas “Año Internacional de la Diversidad Biológica” y en él se espera, por una parte, poder poner en la agenda internacional los problemas de la conservación de los recurso biológicos -más bien

414 complicados en los tiempos que corren-y por otra, comprobar en que medida se han 
cumplido los objetivos fijados para la diversidad biológica en la Cumbre Mundial sobre Desarrollo Sostenible de Johannesburgo, en 2002. En esa ocasión los gobiernos se habían comprometido a "frenar las tasas de pérdida de la diversidad biológica para el año 2010", un objetivo más bien modesto, que contrasta con las metas principales de la cumbre, que eran (nada menos) que acabar con la pobreza en el mundo y lograr cambios en los modelos no sostenibles de producción y consumo. Seis años de crecimiento espectacular (particularmente en China, India, Brasil y otras economías emergentes) y dos de crisis económica más tarde los gobiernos han quizás cumplido en parte sus objetivos de reducción de pobreza, pero descubren que los comedidos objetivos que se habían fijado para la diversidad biológica en el 2002 no se han alcanzado ni se van a alcanzar. La razón es bien sencilla: a pesar de las declaraciones grandilocuentes la protección y uso racional de la diversidad biológica, esta no es una prioridad para ningún gobierno, como tampoco o es el ir hacia un modelo más sostenible de producción y consumo, pura música celestial.

Hay que reconocer con humildad que las sociedades -y los gobiernos- están más preocupadas por las necesidades a corto plazo (el crecimiento económico, el empleo, la obtención de mejoras sociales, la sanidad, la enseñanza y otros asuntos no banales) que por aquellos temas, como el medio ambiente, que sólo pueden plantearse a largo plazo. Nuestro modo de vida prima lo inmediato sobre lo futuro, la satisfacción de necesidades presentes sobre la planificación. Los problemas ambientales (una vez resueltos con más o menos eficacia los más urgentes -como la contaminación de aire que nos rodea o la de los ríos) nos parecen a menudo preocupaciones lejanas.

La reunión de Bonn (incluyo en un recuadro el texto en inglés del mensaje de este encuentro, dado su interés) constataba sobre todo el fracaso de unos compromisos de tipo moral ("evitar la pérdida de la biodiversidad", "limitar la huella del hombre en la Tierra", "evitar la extinción de las especies y la degradación de los hábitats naturales") que los gobiernos y las sociedades rechazan asumir de forma resuelta, aunque lo hagan de boquilla para no quedar mal. El "ecologismo de izquierdas", basado en compromisos morales que el hombre debe o debería tener con el planeta, con los más pobres, con las generaciones futuras y con los otros seres vivos que nos acompañan en esta aventura de la vida está en franca regresión. No ha funcionado. En estos últimos diez años no hemos visto el desarrollo de una economía sostenible. A pesar del éxito parcial de los partidos verdes en las elecciones al Parlamento Europeo y en algunos estados, como Alemania o Francia, éstos siguen siendo minoritarios (en nuestro país sin relevancia) y los grandes partidos se desinteresan de lo ambiental, que empieza a cobrar un tufillo de sesentayochismo caduco a pesar de lo que tiene de auténticamente progresista. En España hemos visto desaparecer el Ministerio de Medio Ambiente en todo menos el nombre, fagocitado por uno de sus antagonistas tradicionales: el Ministerio de Agricultura. La "economía sostenible" no aparece por ninguna parte. El término queda bien en los discursos políticos cuando se propone como el nuevo paradigma económico en tiempos de crisis pero no es creíble cuando se anuncian a la vez ayudas a la compra de automóviles. Es puro aire. 
Frente al fracaso de los compromisos morales se propuso en Bonn lo que algunos bautizan como "ecologismo de derechas", que consiste en dejarse de compromisos con entelequias tan vagas como el planeta y hacer una aproximación fundamentalmente económica a la diversidad biológica, calculando con minucia los beneficios económicos que genera la naturaleza -y la biodiversidad, de modo más amplio- y los muy reales costos a nuestro bolsillo y a nuestro bienestar que conlleva el ignorarla. Se quiere evaluar con detalle el aporte económico de los ecosistemas a la riqueza de las naciones. Al capital y al trabajo hay que añadir en la contabilidad económica todos aquellos valores patrimoniales naturales, que no porque se nos hayan dado gratis no valen nada. Si perdemos agua, suelo, aire limpio, fertilidad de los campos, espacio para la vida, paisajes, playas o bosques para el ocio seremos mucho más pobres, en lo económico como en lo moral.

\section{LOS SERVICIOS DE LOS ECOSISTEMAS, NUEVO LEITMOTIV DE LA BIODIVERSIDAD}

En una época en la que la crisis económica domina el panorama político -por lo menos hasta que, con suerte, se supere en uno o dos años- se estima que la mejor manera de hacer valer la biodiversidad es integrarla en el pensamiento y, sobre todo, en el cálculo económico. La naturaleza nos proporciona una serie de bienes públicos (el agua que bebemos o con la que regamos, el espacio para la agricultura, la ganadería o el disfrute, las maderas de los bosques, los peces del mar, etc.) a los que hay que añadirles los que heredamos de otros seres humanos, las semillas que hacen nuestro pan, los animales silvestres o los que otras personas hace muchos siglos domesticaron e incorporaron a nuestro acervo cultural: la biodiversidad natural o trabajada por la mano del hombre. A muchos de esos elementos es difícil ponerles mercados pues se consideran bienes gratuitos en términos económicos. ¿Cuánto vale una especie que desaparece, una variedad de zanahoria o de patata que puede que nos haga falta mañana? ¿Cuánto valen los genes? ¿Cuánto perdemos en posibles usos futuros cuando se deforestan $100 \mathrm{Km}$. cuadrados de bosque tropical? ¿Y las especies que contiene que ni siquiera hemos catalogado? Estamos quemando bibliotecas de la vida sin haber siquiera leído la mitad de los libros. Lo cierto es que la degradación de los ecosistemas produce pérdidas económicas importantes y muy reales, pero que no parecen entrar en los cálculos económicos al uso. La economía es una ciencia reductora y, a veces, ciega a cosas obvias. El PIB de las naciones no incluye los valores naturales, ni disminuye por las pérdidas de riqueza que puedan ocurrir en ellos la deforestación, la contaminación de los ríos o la pérdida de la fertilidad del suelo, por no hablar de otros valores estéticos como el paisaje o más sutiles e intangibles como los genes de especies que nunca sabremos cómo eran.

Así, los nuevos objetivos que se plantean para la diversidad biológica a partir del año 2010 se orientan hacia una valoración económica más fina de la diversidad bioló416 gica que lleve a las sociedades $-\mathrm{y}$ a los gobiernos- a emplearse seriamente en su 
protección por las consecuencias económicas desfavorables que implica ignorar esos valores. Se pretende hacer una especie de "informe Stern para la biodiversidad" que evalúe las pérdidas económicas previstas de no conservar la biodiversidad, de manera semejante a cómo el informe Stern (2006) evaluó los costes del cambio climático en diferentes hipótesis de acción o falta de compromiso.

Varias organizaciones financian en la actualidad un ambicioso estudio sobre “La Economía de los Ecosistemas y la Biodiversidad" (TEEB en sus siglas en inglés) que pretende "una mejor comprensión del auténtico valor de los servicios de los ecosistemas de modo que se puedan ofrecer herramientas económicas que permitan tener en cuenta de modo apropiado esos valores". El equipo que lleva a cabo el proyecto TEEB, al frente del cual está el economista británico Pavan Sukhdev, está finalizando la redacción del informe, que se publicará en el 2010. El informe tiene recomendaciones precisas para los gobiernos, algunas en particular sobre nuevos parámetros económicos a utilizar en la contabilidad de la riqueza de los estados que tengan en cuenta el valor de lo natural. Debemos esperar, por lo tanto, que entre los nuevos objetivos que se fije el Convenio de la Diversidad Biológica en su reunión del 2010 en Nagoya (Japón) los estados abracen esa nueva visión de la naturaleza y de la biodiversidad como proveedores de servicios al hombre. Queda por ver si esta nueva estrategia, basada en el interés más que en los principios, funciona a medio o largo plazo para obtener los logros ambientales deseados. A juzgar por el fracaso de la explotación internacional de los recursos pesqueros -en los que el interés económico no necesita ser demostrado- no hay mucho espacio para el optimismo.

El encargo del ambicioso proyecto TEEB a un equipo trabajando en Cambridge (eso sí, bajo bandera y en un edificio de Naciones Unidas) no ha sido por azar. En el fondo la visión que impera es la del modelo británico-americano del mundo, su fe en lo económico como valor casi absoluto, su adoración de los mercados, su creencia que el impulso más importante que nos anima a los humanos es el del lucro personal. Hay que poner precios a las cosas porque si no la gente piensa que la Naturaleza y la biodiversidad no valen nada. La gente sólo entiende los precios, el negoci. El mismo modelo que desde hace un año ha mostrado sus límites y nos ha sumido en la mayor crisis económica y financiera desde la de año 1929, queremos que sea convincente para proteger la biodiversidad. ¿Funcionará o habrá que volver a los valores? Oscar Wilde, en frase celebrada, decía que "Un cínico es un hombre que conoce el precio de todas las cosas y el valor de ninguna”. Quizás el apelar a los valores no esté tan pasado de moda como creemos y los gobiernos asuman algún día la responsabilidad que es la suya de velar por aquello que es de todos. De momento los Ministros de Medio Ambiente, ninguneados en los Consejos de Ministros y encuadrados a menudo en el papel secundario de "Ministros Florero" (cuando no desaparecidos) se disponen a cambiar de estrategia y quieren hablar de dineros y precios, a ver si les toman en serio. 


\section{RETOS PARA EL FUTURO EN EUROPA. HISTORIA DE LOGROS Y FRACASOS}

Al margen de cuales sean las negociaciones y nuevos objetivos mundiales para la conservación de la biodiversidad en el marco de Naciones Unidas, no hay que perder de vista que la protección de la biodiversidad no depende exclusivamente de iniciativas globales, sino que está sostenida por unos cambios de pensamiento, de legislación y de práctica que se han desarrollado a nivel nacional en la mayor parte de los países del mundo desde los años 70 y en los que fueron pioneros los Estados Unidos y Europa, muchos de ellos gestados en organismos internacionales.

Es importante saber qué cosas han funcionado y cuales no en los últimos 30 años para saber cuales son los retos que tenemos por delante y evaluar las posibilidades de éxito. Eso unido, naturalmente, al nuevo panorama inédito que va a introducir el cambio climático, quizás el acontecimiento más relevante en la historia de la Tierra de los últimos 200.000 años, pues aunque ha habido muchos cambios climáticos en la historia de este planeta, ninguno ha sido tan brusco.

Las organizaciones europeas, tanto el Consejo de Europa como la Unión Europea han tenido un papel relevante en la evolución de la conservación de la naturaleza y la biodiversidad en el continente, estando a la cabeza de iniciativas consensuadas con los gobiernos, algunas de las cuales se han vistos coronada de éxito y otras menos.

El Consejo de Europa, organismo para el que trabajo (y debo decir aquí que los puntos de vista expuestos en este artículo son sólo míos y en modo alguno los de mi organización) se dio cuenta de forma muy temprana del interés de lo ambiental y fue pionero en proponer instrumentos para la conservación de la naturaleza en Europa. Lo hizo a partir de los años 60, mucho antes de que el medio ambiente se pusiera de moda a raíz de la Conferencia de las Naciones Unidas sobre el Medio Humano de Estocolmo, en 1972, que creó el Programa de Naciones Unidas para el Medio Ambiente (PNUMA). El Consejo de Europa lanzó una campaña intergubernamental muy oportuna, en 1970, llamada “Año Europeo de Conservación de la Naturaleza" de la que salieron dos ideas fundamentales: era necesario crear un sistema coherente de zonas protegidas para Europa (había entonces muy pocas zonas protegidas en el continente) y hacía falta armonizar la legislación que protegía la Naturaleza. La primera idea dio sus frutos (después de algunas tentativas fracasadas) a partir de los años 90 -por legislación específica de la Unión Europea- y ha llevado a la creación de una red europea de zonas protegidas que recibe el nombre de Red Natura 2000 en los países comunitarios y Red Esmeralda en los países fuera de la Unión. La segunda idea se desarrolló a través de la adopción de un nuevo convenio, el Convenio de Berna, de 1979, y dos directivas europeas, una sobre aves silvestres, de 1979, y otra de hábitats y protección de flora y fauna, de 1992.

Estos dos instrumentos, una red de espacios y una legislación moderna prote-

418 giendo la naturaleza han tenido éxito considerable en la protección de la biodiversi- 
dad, ayudando a implantar todo un nuevo sector de actividad en Europa, creándose varios miles de zonas protegidas y estableciendo una verdadera "red de seguridad" de la biodiversidad a la escala de todo un continente. No existe nada parecido en el resto del mundo, ni sistema de zonas protegidas hecho con más rigor. Está todavía en construcción, esperando que se complete en los próximos dos años. Nótese que se van a tardar 40 años de su concepción a su realización, dada la enorme complejidad de la tarea. El sistema funciona razonablemente bien, aunque queda mucho por hacer y a pesar de las presiones del lobby del ladrillo, que en todas partes intenta (en Castilla-León con bastante éxito) saltarse a la torera la legislación europea e internacional que protege los mejores trozos de naturaleza de Europa. La legislación que la mayor parte de los países han adoptado en estos sectores es razonable y eficaz, y se aplica en general bien, aunque quedan algunos cabos sueltos -como el control de las especies exóticas invasoras- de los que hablaré después en la parte dedicada a propuestas.

A pesar del éxito de esas dos iniciativas, que habían acompañado la creación de Ministerios de Medio Ambiente en los años 1980 (España aún tardaría hasta 1996 en crear uno, en inexplicable retraso) se comprobó que las especies seguían desapareciendo fuera de las zonas protegidas por el desarrollo de una agricultura intensiva, una política forestal amiga de la productividad económica y las especies de crecimiento rápido y no de la conservación de los suelos, la flora o la fauna, una urbanización creciente de los espacios litorales y un desarrollo de infraestructuras que fraccionaba el territorio -poniendo barreras a la dispersión natural de las especies y a la conectividad entre zonas naturales- y que sólo respetaba, en las zonas protegidas. Más de un lector recordará polémicas entre constructores de carreteras y pantanos y protectores de la naturaleza, como la polémica autovía de Valencia a través de la Hoces del Cabriel, dónde el antiguo Ministerio de Obras Públicas y Urbanismo, que curiosamente acumulaba sin ejercerlas las competencias en medio ambiente, se empeñaba en hacerla pasar por lo que hoy es un parque natural. Se pensó entonces, a escala europea en lanzar un "Segundo Año Europeo de Conservación de la Naturaleza, 1995" que ponía el énfasis, 25 años después del primero, en la conservación de la biodiversidad fuera de las zonas protegidas, en la necesidad de integrar naturaleza y desarroIlo, influido sin duda por la Cumbre de la Tierra de Río de 1992 que había visto nacer el Convenio de la Diversidad Biológica. La idea es que no bastaban las zonas protegidas, que había que integrar estas mejor entre sí funcionalmente, restaurar las zonas degradadas, evitar que las zonas protegidas se convirtieran en "catedrales en medio del desierto", del desierto de vida en que se estaban convirtiendo los paisajes rurales europeos, sacrificados al productivismo y a la denostada PAC, la Política Agraria Comunitaria. Esta política vertía millones de euros en los campos de Europa (sobre todo en Francia) empujando a los agricultores a unas producciones altísimas, un uso desproporcionado de fertilizantes y pesticidas y una desaparición de todo atisbo de lo natural, los pequeños setos, los bosquetes, los cultivos menos rentables.

Se lanzó entonces esa idea de "integrar la biodiversidad en las otras políticas" e incluso se propuso una nueva "Red Ecológica Paneuropea" que integrara las zonas 
protegidas con otras de menor valor ecológico en un conjunto armonioso que pudiera servir de base a la ordenación más racional de los espacios rurales y las costas de Europa. En la Conferencia de Ministros "Un Medio Ambiente para Europa" (Sofía, Bulgaria, 1995) el Consejo de Europa llevó una estrategia para la aplicación del Convenio de la Diversidad Biológica en Europa, con el complicadísimo nombre de "Estrategia Paneuropea para la Diversidad Biológica y de Paisajes", (PEBLDS en sus siglas inglesas) que fue adoptada con entusiasmo por los ministros pero dio posteriormente pocos frutos prácticos (al margen de unas pocas conferencias llamadas "Biodiversidad para Europa") y creó muchas burocracias complicadas.

El problema esta vez no era que las ideas no fueran buenas o acertadas, era que unos ministros de medio ambiente de poco peso político (excepto en algún país escandinavo) lograban poner en marcha políticas de control de contaminantes, de impactos de algunas actuaciones o de protección de espacios singulares, pero eran completamente incapaces de lograr cambios significativos en las políticas que de verdad mellan la biodiversidad: la políticas agrícolas, las de transporte y construcción de infraestructuras, las de pesca, las industriales, las de urbanismo, las de energía, las de comercio, las políticas del agua (véase como muestra en qué han quedado en España los límites ecológicos a los trasvases y las políticas de mantenimiento de la integridad ambiental de las cuencas hidrográficas una vez que las competencias han pasado al departamento responsable de la políticas agrícolas o, para algunas cuencas, a las comunidades autónomas). Sin un arbitraje favorable del jefe del ejecutivo en casos de conflicto, los ministros de medio ambiente no logran sus objetivos en biodiversidad, cueste o no cueste al país a largo plazo ignorar las leyes de la naturaleza y del sentido común.

Como muestra vale un botón: desapareció la anchoa del Cantábrico mientras íbamos a Bruselas a pedir que la Comisión nos dejara pescar las anchoas que no había, con el entusiasta apoyo de Francia. No se escuchaba a los científicos. Cuatro años de veda no han bastado seguramente para reponer una pequeña parte de lo que era una vez una pesquería boyante. Nadie se responsabiliza de las miles de empleos perdidos en los barcos y la industria conservera, ni de que los consumidores nos hayamos quedado sin ese excelente producto. Con el atún rojo en el Mediterráneo vamos por el mismo camino, hasta el punto de que se está pensando en ponerlo en las listas de especies amenazadas.

\section{LO QUE QUEDA POR HACER: ALGUNAS PRIORIDADES PARA LA BIODIVERSIDAD EUROPEA}

He escogido, de los múltiples retos delante de nosotros, unos pocos para ilustrar algunas de las tareas pendientes en Europa. En esos asuntos estamos intentando trabajar las instituciones europeas, tanto en Bruselas como en Estrasburgo. No son todos los desafíos a los que nos enfrentamos, pero pueden dar una idea del mucho trabajo pendiente en biodiversidad. El fracaso relativo de los compromisos de los

420 gobiernos no debe hacernos perder de vista que la tarea es de una gran importancia 
para el bienestar de las generaciones futuras, piensen lo que piensen las actuales clases políticas, ensimismadas en sus caducos modos de ver el mundo.

\section{El cambio climático}

Puede que sorprenda que, entre los retos a los que se enfrenta la biodiversidad biológica en nuestro continente, mencione en primer lugar el cambio climático cuando hay otras presiones y amenazas más acuciantes. Para muchas personas el cambio climático es una preocupación para el futuro, algo de lo que nos debemos ocupar en el presente, cierto, pero que sólo se hará sentir dentro de muchas décadas. Pensamos que afectará a otras generaciones, no a la nuestra y que quizás los que nos sucedan en este río infatigable de la vida, ya se hayan acostumbrado a los calores que se anuncian. Se nos pide ahora que hagamos grandes inversiones ( $i c o n$ la deuda inmensa que estamos generando en la resolución de esta crisis!), peor, que cambiemos nuestro modo de vida produciendo menos gases de efecto invernadero para evitar que, al fin del siglo XXI la subida de la temperaturas media del planeta no sea mayor de 1,5 ó 2 grados y no los 4 ó 6 que pueden venir si no se toman medidas. A nosotros, que seguramente en año 2100 hayamos dejado ya de tener calor. A esa visión de "problema para el futuro" contribuye en no poca medida el haber enfocado las discusiones sobre cambio climático casi exclusivamente en el calentamiento y no en otros factores de tanta o mayor importancia, como son los cambios de regímenes de lluvias. Se nos dice que a final de siglo París tendrá el clima de Burdeos -y quizás sus caldos- a no ser que no hayamos echado bien las cuentas y tenga el clima de Sevilla. Cada noticia que aparece en la prensa sobre este asunto es más alarmante. El último y cuarto informe del IPCC, el Panel Intergubernamental para el Cambio Climático-, publicado en 2007, parece más bien timorato en sus predicciones. Los científicos dicen dos cosas muy claras: la primera que el cambio climático ya está aquí y la segunda que va mucho más deprisa de lo que se creía hace sólo dos años.

El cambio climático es, como decía arriba, un acontecimiento sin precedente en la historia biológica de Homo sapiens y en la del propio período cuaternario. Este periodo interglaciar en el que prosperamos como especie va a durar mucho más que los precedentes y va a hacer mucho calor. Si dentro de un instante (a escala evolutiva) París puede tener el clima de Sevilla, ¿Qué clima tendrá Sevilla? ¿El de Tamanrasset? El último período interglaciar fue hace 120.000 años y esta crisis climática de origen humano muchos investigadores la asocian a un primer calentamiento global producido hace 10.000 años por las quemas de bosques para agricultura y ganadería al comienzo del Neolítico, de modo que esta sería, en puridad, la segunda crisis climática de la que nuestra especie es responsable, más grave que la primera pues hemos quemado en 150 años unas cantidades ingentes de carbón y petróleo y producido, además de dióxido de carbono, otros gases con efecto invernadero.

Las especies están ya cambiando sus áreas de distribución. En Europa muchas mariposas proliferan ya más de trescientos kilómetros al norte de su distribución 
"tradicional" o han "subido" doscientos metros de altitud en las montañas. Las frías temperaturas invernales que limitaban su distribución se han suavizado. Muchas especies de plantas están adelantando sus periodos de floración (de 2 a 8 días de avance por década, según distintos estudios) o retardando la caída de las hojas en otoño. La masa de datos recolectada es abrumadora. El cambio climático va a modificar también los regímenes de lluvias. Donde antes había humedales no lloverá lo suficiente para mantener sus ecosistemas característicos. Muchas zonas de bosque mediterráneo pueden convertirse en estepas áridas o desiertos. Las especies exóticas invasoras verán aumentar su distribución y efecto sobre las especies autóctonas. La migración de las aves puede cambiar radicalmente. Muchas especies dejaran de migrar. Una extinción masiva está en marcha y las medidas de adaptación de la diversidad al cambio climático hay que tomarlas ahora, no dentro de veinte años. Nuestro continente se verá particularmente afectado por esta nueva "sexta extinción" planetaria (la última fue hace 65 millones años), la primera causada por la acción humana, porque, como señala el informe del IPCC (2001) "el potencial de adaptación de los sistemas naturales es generalmente bajo" en parte porque "Europa es predominantemente una región de hábitats naturales y semi-naturales fragmentados en un paisaje agrario altamente urbanizado". Lo tenemos difícil.

En las negociaciones sobre el cambio climático que han tenido lugar en Copenhague a final del 2009 se ha empezado a hablar más de adaptación, pero otra vez la economía "tradicional” gana por goleada a la economía basada en la Naturaleza. Se quieren dar compensaciones y buscar soluciones para sectores como las estaciones de esquí, que cerrarán sus negocios por falta de nieve, pero no se rediseña el sector forestal, no se dan pistas -ni recursos- para saber cómo se ha de cambiar la gestión de las zonas protegidas, la gestión de las especies. A pesar de que la biodiversidad puede contribuir en buena medida a la atenuación del cambio climático, pues plantaciones de nuevos bosques podrían actuar de "sumideros" donde retener carbono, sólo de forma muy marginal se da cancha a lo natural dentro de los esfuerzos de atenuación en las negociaciones climáticas.

En cualquier caso hay que darse cuenta que el cambio climático no sólo está ya aquí, sino que es imparable. Lo que se discute ahora es si será moderado o insoportable para nuestra civilización. Tendrá consecuencias muy desfavorables para la biodiversidad sobre la Tierra. La velocidad del cambio hará que muchas especies pierdan gran parte de su variabilidad genética, al no lograr adaptar su ritmo de colonización de nuevos hábitats favorables a la pérdida brusca de los sitios en los que ahora viven, quedando más expuestas a la extinción.

Europa debe abrir el camino y mostrarse a la altura de las circunstancias en este momento, negociar seriamente para reducir las emisiones y, sin esperar más, ir elaborando políticas serias de adaptación de la biodiversidad al nuevo reto. Dos zonas del continente son particularmente frágiles a los cambios climáticos, el ártico y el medi-

422 terráneo. Estamos acostumbrados a asociar el cambio climático con la reducción 
drástica del casquete polar y de la banquisa ártica, que puede llegar a desaparecer completamente en verano en un futuro no muy distante. El deshielo de la banquisa, acelera el cambio climático pues su color blanco contribuye a reflectar a la atmósfera buena parte de la radiación, lo que no ocurre con el agua del océano. El oso polar está condenado a largo plazo por pérdida de su hábitat. Sin embargo el ártico es una zona poco poblada (menos de 4 millones de habitantes) y su biodiversidad una de las más bajas del planeta. La región mediterránea, sin embargo, tiene una población muy alta (unos 300 millones de habitantes en las dos orillas del mar) y contiene las zonas de más alta biodiversidad de nuestro continente. Las pérdidas de biodiversidad y los conflictos medioambientales, por ejemplo por competencia por agua, serán enormes. Eso por no mencionar las posibles pérdidas de recursos agrarios por erosión, acidificación, aumento de incendios forestales, factores todos que contribuirán a fomentar los flujos migratorios entre África y Europa.

Una buena parte de la biodiversidad europea se encuentra en los macizos montañosos y en las islas. Estos ecosistemas han conocido un aislamiento geográfico o ecológico que ha llevado a la diferenciación de formas vivas que evolucionan en nuevas especies características (especies "endémicas", aquellas que son únicas de una región). A pesar de su riqueza biológica ha habido pocas políticas europeas o nacionales específicamente dirigidas a estos ecosistemas de montaña o insular. El aislamiento geográfico o ecológico hace más frágiles a estas especies frente al cambio climático. Si otras especies del continente tienen la posibilidad de migrar hacia el Norte (si no se extinguen antes) eso no es posible en las islas o en las zonas de montaña. No es siempre fácil que una especie de Sierra Nevada dé el salto a la Ibérica o al Pirineo, particularmente si se trata de pequeños anfibios (que sufren, además una crisis global) o reptiles. La Agencia Europea del Medio Ambiente avanza en un estudio que hasta un $60 \%$ de las especies de la montaña mediterránea pueden extinguirse, lo que juzgo un poco exagerado pero da una idea de la magnitud potencial de los riesgos sobre esa frágil biodiversidad. Muchas especies de montaña o endemismos insulares pueden convertirse en "especies huérfanas", es decir, especies cuyo ecosistema ha desaparecido pero que todavía se conservan en cautividad o bancos de genes. Ahora existen unas pocas especies huérfanas en el mundo (por ejemplo una paloma forestal de una isla del archipiélago de las Seychelles en la que ha desaparecido el bosque). El cambio climático puede producir cientos de ellas. Ahora es el momento de decir qué podemos hacer con ellas o de decidir, por ejemplo, si se debe o no aceptar la "migración asistida" (Ilevar artificialmente especies a la zonas donde se supone que el nuevo clima les puede ser favorable). La pregunta no es puramente retórica. En los Estados Unidos, que siempre nos Ileva un adelanto de unos años en ideas sobre conservación de la naturaleza, la migración asistida tiene ya sus partidarios y sus éxitos. Además es muy popular entre ciertos botánicos y entomólogos. El movimiento voluntario ya está moviendo una especie amenazada de árbol de Florida a Carolina del Norte, de donde la "habían echado" las glaciaciones. Mover animales y plantas es una vieja manía humana. También nuestros vecinos franceses introdujeron la marmota en el Pirineo, "corrigiendo" otra extinción debida a las glaciaciones. 
Europa debería dar normas claras de cómo gestionar la biodiversidad frente al cambio climático para evitar extinciones y todos juntos debemos encontrar los recursos necesarios para esta ingente tarea. También tendrá que cambiar nuestra manera de seleccionar y gestionar las zonas protegidas, pero de eso se hablará más abajo.

\section{La integración de la biodiversidad en políticas sectoriales}

La agricultura ocupa una mayor parte de la superficie no urbana de Europa, junto con la ganadería y la actividad forestal. El modelo europeo de los últimos cuarenta años, es decir, el fomento de la agricultura intensiva con fondos públicos a fin de mantener artificialmente ocupado el medio rural ya no se justifica ni desde el punto de vista económico, ni moral, ni ambiental. Los alimentos no son ni puede ser ya un recurso de interés estratégico en un mundo globalizado. Esas ayudas a los agricultores (que deberían transformarse en ayudas a un mundo rural más ecológico y sostenible) no sólo introducen un factor de injusticia a nivel planetario, al impedir el acceso en condiciones de igualdad de los agricultores pobres a los mercados europeos (y americanos o japoneses), también llevan a una degradación de los paisajes agrarios, a una contaminación de los ríos (que luego habrá que limpiar con más gasto público) y a unas pérdidas enormes de diversidad biológica. El modelo está caduco, es gravemente lesivo para los intereses de la mayor parte de los europeos, consume cantidades ingentes del presupuesto de la Unión para beneficiar a muy pocos (que ni siquiera lo agradecen), es anti-ecológico y debilita las posturas europeas las negociaciones comerciales mundiales de comercio. Si lo hemos mantenido tanto tiempo es por las presiones de los países favorecidos, sobre todo Francia, pero también España y otros. La Unión Europea no tendrá credibilidad en sus políticas ambientales sólo por obligar a los países a crear zonas protegidas, sino por cómo genere una nueva PAC que respete la naturaleza y cree nuevas zonas de biodiversidad en el paisaje agrario común. Suiza (fuera de la Unión) ya está condicionando sus ayudas a los agricultores a que dejen una parte de cada explotación en uso "ecológico", intentando crear una fina red de teselas de naturalidad y biodiversidad en los paisaje agrarios. Hay que vencer las enormes resistencias de Francia y otros países a seguir "ordeñando la vaca" comunitaria ad aeternum so pretexto que cuando se diseñaron esas políticas la alimentación era importante (y aún más importante que Alemania corriera con la mayor parte del gasto y Francia hiciera caja).

Se nos dice que el fin de esas políticas llevará a un abandono del campo, pero eso puede ser compensado por otro tipo de ayudas al medio rural y, en último caso, no hay nada de malo en un campo donde la naturaleza avance. Esos territorios se van haciendo raros -y por tanto valiosos- en Europa y pueden ser desarrollados a través del turismo rural u otras actividades nuevas. La provincia de Teruel, por ejemplo, tiene menos de 10 habitantes por kilómetro cuadrado y eso no supone ningún problema grave (el abandono político, sí). Ciertamente sus habitantes disfrutan de mucho más

424 espacio que los 400 holandeses que se tienen que empaquetar en cada kilómetro 
cuadrado de los Países Bajos rodeados de carreteras, casas, vacas en explotación intensiva y campos de tulipanes.

Europa podría asumir un liderazgo ambiental, desmontar la agricultura intensiva, fomentar nuevas políticas agroambientales y ser decisiva a la hora de concluir la ronda de Doha de la OMC, lo que nos ayudaría a romper el egoísmo e insolidaridad que en tiempos de crisis hace que los gobiernos se dejen engatusar por las sirenas del proteccionismo. Nadie sale solo de una crisis semejante.

De un modo parecido la producción forestal debe hacerse más ecológica de lo que lo es ahora, pues es fundamental para el mantenimiento de la biodiversidad. Hay 10 millones de kilómetros cuadrados de bosque en Europa, que representan una tercera parte del continente y de los que la parte europea de Rusia supone un $80 \%$. Ya la Conferencia Ministerial sobre Protección de los Bosques en Europa ha adoptado desde el 2003 principios para la gestión forestal sostenible, y la multiplicidad de los usos del bosque, pero la práctica dista mucho de los principios. Sigue sin haber una proporción aceptable de zonas forestales protegidas y en muy pocos lugares se da la prioridad a la conservación de los recursos vivos sobre la producción de madera. La superficie forestal aumenta poco a poco en Europa, pero la mayor parte de los bosques en la Unión Europea son relativamente jóvenes, siendo escasos los bosques antiguos, aquellos que son más ricos en biodiversidad.

Otras políticas sectoriales (el transporte o la energía, por no hablar del urbanismo desatado que se abate como plaga bíblica sobre innumerables lugares de interés natural) han fallado en integrar el respeto a la diversidad biológica en sus actividades. Vemos, por ejemplo, carreteras cortar zonas de naturaleza valiosa sin que se hagan las necesarias medidas de corrección que permitan el paso de la fauna, la cual va quedando progresivamente encajonada en reductos cada vez más pequeños y desconectados, empujando las especies a la pérdida genética y la extinción. La red de carreteras de Europa se va densificando cada vez más, a medida que la naturaleza se encoge. Haría falta un plan ambicioso de "desfragmentación" del territorio, que permitiera restaurar las conexiones vivas y funcionales entre paisajes separados por infraestructuras o zonas de agricultura intensiva. Para ello es necesaria una restauración ecológica de muchas zonas y una ordenación real de los usos del territorio, no ficticia como ocurre en la actualidad, donde cada municipio hace de su capa un sayo y el ladrillo abre sus erizadas flores minerales a gusto del constructor del lugar, en un ejemplo de simbiosis de intereses privados con otros inconfesables pero públicos.

En el campo de las energías renovables, se han realizado importantes avances en los últimos diez años y cada vez vamos a tener una mayor proporción de nuestra energía (fundamentalmente la eléctrica) de fuentes renovables. El problema es que muchas de las nuevas instalaciones, en particular las centrales eólicas, causan problemas ambientales, afectando el paisaje, la biodiversidad y son fuente de ruido y molestias para personas que vivan en su cercanía (además de ver bajar espectacularmente el precio de sus casas). Las centrales eólicas afectan a la avifauna, a los murciélagos y, 
en las instalaciones marinas, a los cetáceos, a causa de los ruidos. La estructura colosal de sus molinos, que a veces sobrepasan 100 metros de altura, los asemeja a los gigantes contra los que se rebelan los nuevos quijotes: no es de extrañar que el pasado mes de junio 340 asociaciones ecologistas y ciudadanas europeas pidieran una moratoria de su construcción en el continente hasta que haya normas claras de dónde se pueden instalar y dónde su impacto ambiental es insoportable. En el sur de Francia a menudo se colocan en roquedos y otras zonas de interés botánico. En España han florecido como hongos en sitios venteados, como en la zona del estrecho donde también se concentran las migraciones de aves entre Europa y África, con el consiguiente peligro para estas últimas. En España ya hay 670 parques eólicos, cuya construcción entusiasta por la iniciativa privada sólo ha sido frenada al final por el congelamiento de las ayudas públicas, pues al final se trata de una industria subvencionada y no había dineros en las arcas ya muy menguadas del estado para financiar tanta central como se había previsto. Lo peor es la ausencia de criterios claros sobre su ubicación. No se respetan las zonas Natura 2000 designadas para las aves ni se hacen informes de su impacto sobre la biodiversidad.

Es urgente que, desde las instancias europeas, se hagan normativas o directrices para la ubicación de centrales eólicas a fin de evitar que se construyan donde los impactos ambientales (o sobre los paisajes o el patrimonio cultural) se consideren inaceptables. Un grupo de trabajo específico de la Comisión Europea lleva más de dos años trabajando en ello, aunando los esfuerzos de las direcciones generales de medio ambiente y energía y transporte, sin resultados prácticos por el momento. Se debería dar una normativa rápidamente, no cuando ya se hayan construido todas las centrales que los presupuestos públicos tengan capacidad de financiar. Habrá que estudiar también mejor la posible instalación de parque eólicos marinos lejos de las costas, donde su impacto ambiental sea menor

\section{Cambiar el modelo de zonas protegidas}

En los próximos años debe hacerse un esfuerzo particular por mejorar el sistema europeo de zonas protegidas, que ha sido uno de los grandes logros de los últimos veinte años pero que dista todavía mucho de ser satisfactorio. Es cierto que la designación de sitos de interés comunitario dentro de la Red Natura 2000 en el marco de la Directiva Hábitats y, paralelamente, la designación de Áreas de Especial Interés para la Conservación (Red Esmeralda, en los países que no son Estados Miembros de la Unión Europea) ha supuesto un avance importantísimo. Sabemos ahora con gran precisión donde están los lugares de mayor interés ecológico del continente y grosso modo la biodiversidad que contienen. Falta, sin embargo, que estos sitios sean el objeto de planes específicos de acción, que no sean "parques de papel” cuya protección no se vea acompañada de una mejora de sus valores y de la puesta en marcha de unas políticas de restauración y manejo que hagan de estos territorios la verdadera estructura verde del territorio. Dejo de lado el hecho de que, en muchos países, esas zonas

426 designadas cubren verdaderamente un porcentaje muy pequeño del territorio y se 
ven aislados entre grandes zonas agrarias de mayor o menor grado de alteración. El sistema de áreas protegidas, pues, de be ser reforzado y completado con más y mejor legislación y más acción sobre el terreno.

Otra tarea muy importante en ese campo es la de encuadrar mejor las zonas protegidas con el resto del territorio, creando los corredores necesarios para que puedan establecerse conexiones funcionales entre las distintas áreas que permitan, entre otras cosas, la migración de las especies y el establecimiento de continuos genéticos entre distintas poblaciones existentes en zonas protegidas. En el fondo se trata de "repensar" el modelo existente, no basándolo como se ha se ha hecho hasta ahora, en la declaración de áreas protegidas cuando había especies o ecosistemas raros o de interés, sino pasando a hacer un análisis más profundo del territorio que proteja los procesos ecológicos que están en el origen de esas especies y ecosistemas. En el Consejo de Europea se lanzó en los años 90 la Red Ecológica Paneuropea, que pretendía construir una red más amplia de espacios integrando los espacios protegidos (las actuales zonas Natura 2000, Esmeralda y otras), uniéndolos por corredores ecológicos e identificando otros espacios de relativo valor -como, por ejemplo, zonas rurales bien conservadas- que permitieran dar a todo el conjunto una unidad espacial. Se pretende que las redes ecológicas no sean sólo virtuales, sino que tengan una realidad y una continuidad física en el territorio, es decir, que estén de verdad conectadas. Hay un límite al número y extensión de las zonas protegidas que se pueden declarar sin afectar a la economía o a la resistencia de los usuarios del medio rural, de modo que para lograr la protección de esas nuevas zonas de interés hay que usar políticas agroambientales o de ordenación del territorio. Las políticas puramente de conservación no valen. El problema, como se comentó arriba, es que para que esas redes ecológicas se creen de verdad es necesario avanzar en la integración de la biodiversidad en otras políticas, algo en lo que se va acumulando mucho retraso. Hay que proteger la biodiversidad fuera de las zonas protegidas si no queremos ver éstas “implosionar" por aislamiento genético y perder su biodiversidad. La restauración de hábitats degradados es otra prioridad en eses sentido. Esas ideas están también tomando cuerpo en un proyecto de la Unión Europea llamado "Infraestructuras verdes para Europa”, en el que se sostiene que para la riqueza y prosperidad de Europa hace falta la creación o mantenimiento de una base ecológica (o infraestructura verde) del mismo modo que se construyen infraestructuras de carreteras, eléctricas, redes ferroviarias de alta velocidad, se implanta la televisión de alta definición o se desarroIlan las redes informáticas de alta capacidad, etc. La infraestructura verde nos daría otro tipo de servicios en los que a menudo no pensamos. Es buena idea, pero no será fácil llevarla a la práctica.

Si ya es difícil hermanar la gestión de las zonas protegidas con el resto de actividades que se desarrollan en su entorno, va a ser incluso más complicado repensar todo el sistema debido al cambio climático. Ahora que, por fin, vamos a tener una verdadera red europea de zonas protegidas, cuarenta años después de haberlas concebido, no parece que sea el momento adecuado para rediseñar el sistema, pero lo cierto es que el cambio climático obliga a replantarse las bases sobre las que hemos 
elegido esas zonas. Ahora se protegen unas zonas naturales porque contienen hábitats interesantes (por ejemplo humedales) o porque en ellas se encuentran especies valiosas o raras. El sistema actual está pensado para un clima estable, no para uno que cambia a la velocidad con que lo hace el nuestro, así que no nos queda más remedio que ver de que forma variamos o completamos el sistema actual para responder al reto de proteger al biodiversidad en un marco de crisis climática. Un ejemplo: para final del siglo XXI se anuncian precipitaciones de hasta el 50\% más bajas par muchas zonas de la Península ibérica, a la vez que las temperaturas podrán a llegar a subir entre 2 y 4 grados. Es obvio que muchos humedales que ya tienen problemas de conservación (por la mala gestión del agua) como la Mancha húmeda sencillamente no tendrán agua a final de siglo. ¿Qué haremos con el Parque Nacional de Daimiel cuando sea un secarral? (apunto en passant que ya va camino de ello por la explotación insostenible del acuífero que nutre el parque, ante la indiferencia general y las soluciones de parcheo de trasvases a un pequeña parte de su extensión original). ¿Deberemos quitarle la protección? ¿Se propondrá como nuevas tierras de cultivo? ¿Y qué hacer con una zona de la red Natura 2000 que haya sido declarada interés para una especie de ave que haya migrado al norte siguiendo climas más propicios? ¿Deberá sacarse de la red? Está claro que el sistema no podrá seguir siendo el mismo. Habrá que adaptarlo a los nuevos parámetros que trae el cambio climático. Pienso que sería bueno definir una especie de "reserva de suelo para naturaleza" (los portugueses tenían antes una figura parecida, con el nombre poco afortunado, por confuso, de "reserva ecológica del territorio" que comprende, en lo esencial, todas aquellas zonas que no eran urbanas o agrícolas y que deberían tener un uso fundamental par la protección de los ciclos vitales y el medio ambiente.) Ese nuevo espacio, que encaja bien con el concepto de "infraestructura verde" podría definirse en función no tanto de las especies o de los hábitats presentes, sino del grado de naturalidad del territorio. Así se podrían declarar y proteger unos "espacios de gran naturalidad y pequeña influencia humana" que serían conservados independientemente de las especies o hábitats que contuvieran, pues estos cambiarían con el tiempo siguiendo las pautas climáticas, pero tendrían el interés de mantener procesos ecológicos, al margen de las especies y los hábitats. La presencia de especies o hábitats raros no serían ya los únicos criterios para la definición de espacios a proteger. Ya veremos si estas ideas se abren paso en los próximos diez años y permiten completar las redes actuales con otras nuevas zonas, manteniéndose ese tejido vivo del que dependemos.

\section{No olvidar las especies}

Aunque en conservación de la biodiversidad es conveniente poner el énfasis en la conservación adecuada de los espacios naturales y en su integración armoniosa con el resto de la trama territorial, esa ambición no debe hacernos olvidar las especies. La supervivencia de muchas especies no se logra únicamente a través de políticas de espacios protegidos, aún siendo estas esenciales. Hay muchas especies en una situación tan crítica que requiere medidas especiales de manejo y gestión que pue-

428 den incluir en muchos casos acciones de conservación "ex-situ". Es el caso de muchas 
especies vegetales cuya variabilidad es necesario conservar en bancos de germoplasma y que necesitan también de trabajos "activos" de reproducción en invernadero para su posterior plantación en lugares idóneos con el objeto de hacer nuevas poblaciones que disminuyan las probabilidades de extinción en la naturaleza. Para algunas especies muy amenazadas, como el lince ibérico, no basta su conservación en le campo, por bien que se haga y por muchos recursos que se le dediquen. Hace falta continuar con el programa tan exitoso de cría en cautividad. Toda la población, en cautividad y en el campo se maneja ahora como una sola metapoblación, sacando individuos del campo para su cría en cautividad para posteriormente alimentar con individuos cautivos poblaciones salvajes y, crear otras nuevas, intentando maximizar la diversidad genética que es el mejor seguro de supervivencia de esa especie cuando se recuperen las poblaciones de sus presa, el conejo. También hay que considerar que muchas especies de interés, como los lobos, tienen áreas de campeo muy grandes y que no habría modo de mantenerlos sólo en zonas protegidas, por lo que debe haber una políticas europeas que fomenten la convivencia pacífica entre, por ejemplo, grandes carnívoros y el hombre, ya que estos van a vivir en gran medida en hábitats agrarios relativamente humanizados. No es fácil que los ganaderos acepten de buen grado los daños que les causan especies que se alimentan del ganado y podrían fácilmente eliminar si no estuvieran protegidas. Aquí debe primar el sentido común, es decir la socialización de las pérdidas que esos animales causen en las explotaciones ganaderas y el mantenimiento de sus poblaciones en un nivel en el que se minimicen los conflictos y se asegure a la vez que esas especies estén en un estado favorable de conservación. Los maximalismos (de protección o de control de depredadores) pueden generar conflictos que no interesan a nadie. La recuperación espectacular del lobo en Francia, España, los países escandinavos y otras zonas del continente ha generado muchos problemas de aceptación de este cánido, lo que no es de extrañar, pues los métodos de gestión del ganado se habían adaptado a la falta de lobo y los cambios hacia fórmulas antiguas son siempre complicados. En Europa hay espacio para un pastoralismo respetuoso de la naturaleza y para los grandes carnívoros. Tanto el Consejo de Europa como la Comisión Europea han estado en los últimos años trabajando en ese sentido, dando recomendaciones para la gestión de poblaciones de grandes carnívoros (oso, lobo, linces, glotón) y animando a los estados a colaborar en la gestión de poblaciones transfronterizas, con éxito variable. Es de destacar en este contexto el papel fundamental de asesoramiento de un grupo de científicos especializados en estos animales, vertebrado en un grupo de la Unión Internacional para la Conservación de la Naturaleza y de los Recursos Naturales (UICN) Ilamado "Iniciativa Europea para los Grandes Carnívoros", LCIE en sus siglas inglesas.

También hay muchas otras especies que necesitan que se pongan en marcha los planes de acción o de recuperación que han sido elaborados, como el visón europeo, que cada vez ve disminuir más su área de distribución (excepto en España, donde parece adaptarse en las últimas décadas a paisajes mediterráneos) o la foca monje, cuyo veloz proceso de extinción entre los años 40 y 80 parece haberse frenado pero que sigue con todas las alarmas encendidas. También las tortugas marinas, muy castigadas por la ocupación por segunda residencia de sus playas de puesta y por un 
número enorme de capturas accidentales en redes de pesca y palangres, deben ser objeto de especiales medidas de conservación, igual que un par de docenas de especies de aves cuyas poblaciones están en estado crítico y no pocas especies de invertebrados, a los que por desgracia se les dedica poca atención, honor que comparten con otro grupo de mamíferos que también tiene varias especies amenazadas: los murciélagos. Desde las instituciones europeas se han elaborado y financiado muchos planes de acción pero no siempre se ve a los gobiernos muy dados a su aplicación. La dispersión de competencias entre autoridades nacionales y regionales (incluidos los "länder" y las comunidades autónomas) no siempre facilita la coordinación europea.

\section{Combatir las especies exóticas invasoras}

Un elemento fundamental de acción en el futuro debe ser el control de las especies exóticas invasoras. Estas encuentran en la globalización su panacea. Al haber aumentado de modo exponencial en los últimos años el comercio, el transporte de mercancías y el movimiento de personas, se está produciendo un verdadero aluvión de nuevas especies en Europa y la dispersión de especies exóticas dentro de Europa. Es cierto que la mayor parte de las especies exóticas que llegan a territorios nuevos no causan mayor problema, pero una pequeña proporción (que puede al final llegar a ser muchas especies) consigue establecerse y algunas pueden causar problemas serios a los ecosistemas o a las especies autóctonas. Se considera que las especies exóticas son la segunda causa de extinción de especies en el planeta, (después de la destrucción o deterioración de los hábitats naturales) sobre todo por como afectan a los endemismos insulares. Algo más de la tercera parte de la flora de cada isla europea (por termino medio) está formada por especies que no son de allí. Cada año se establecen en Europa casi 15 especies nuevas de flora (lo que supone casi 1500 por siglo). Muchas de las especies exóticas no llegan por azar, sino son traídas ex profeso para su uso en jardinería, en agricultura, en arboricultura, en los acuarios. Los cazadores y pescadores son muy dados a introducir especies nuevas para su actividad, algunas de las cuales son deletéreas para las especies autóctonas o traen parásitos o enfermedades que estas no resisten (como la llamada "plaga" del cangrejo, que nos dejó sin aquellos magníficos y deliciosos cangrejos nuestros que yo recuerdo de los años 60 con gastronómica fruición y que hoy son casi historia). Muchas otras especies llegan en embalajes, en el suelo que acompaña a las plantas de invernadero, como semillas mezcladas con otras semillas comerciales "inocuas", en aguas de balaste, pegadas a embarcaciones de recreo (como el famoso "mejillón cebra") o de muchas otras maneras (serpientes traídas como animales exóticos de compañía, por ejemplo y que luego escapan y hacen estragos en el campo).

Es necesario que se explique mejor a la gente los efectos nefastos de las especies exóticas sobre la biodiversidad local y, a veces, sobre la salud. La falta de concien430 ciación hace que se traigan muchas especies que no deberían entrar nunca en nues- 
tro continente. Un supermercado, por ejemplo, regalaba a sus clientes al hacer la compra en España una planta acuática de las Américas, el jacinto de agua o "camalote" (bellísima, por cierto) sin saber que una invasión de esta bella plaga había cubierto decenas de kilómetros del río Guadiana, con un coste de eliminación superior a los 15 millones de euros.

Los organismos europeos han hecho recomendaciones para limitar el movimiento de estas especies. En particular el Consejo de Europa adoptó en el 2003 una Estrategia Europea para la Especies Exóticas Invasoras que ha servido a muchos gobiernos para tomar conciencia del problema y poder vertebrar una respuesta coordinada. La Comisión Europea ha registrado casi 15.000 especies exóticas presentes en Europa, incluidas en una base de datos llamada DAISIE y está poniendo ahora los cimientos para un sistema de alerta temprana y actuación rápida una vez que se identifica una nueva invasión. La Organización Europea y Mediterránea para la Protección de Plantas (EPPO) ya ha desarrollado un sistema para evaluar el riesgo ambiental de nuevas plantas que llegan y, junto con el Consejo de Europa, ha desarrollado un "Código de Conducta sobre la Horticultura y las Plantas Exóticas Invasoras" que quiere hacer colaborar a la industria en la prevención de nuevas introducciones y en el control de la dispersión de especies que se comercializan. La Comisión Europea, después de lanzar una vasta encuesta entre los medios científicos y ciudadanos, ha adoptado un documento político (una "comunicación" en el lenguaje misterioso y tecnocrático de Bruselas), pero lo que hace falta es una legislación europea ("directiva" o "reglamento") que ponga coto al comercio de estas especies. Bajo la regla de que ya no hay fronteras comerciales intracomunitarias para los bienes, se permite ahora la libre circulación de estas especies exóticas invasoras sin tener en cuenta sus daños ambientales. Es de prever, además, que muchas especies exóticas que están ya presentes en Europa pero cuya expansión estaba controlada por nuestros rigurosos inviernos, puedan hacerse invasoras al amor (cálido) del cambio climático. También es más que previsible que en un futuro no muy lejano muchas especies invasoras que llegaban y no lograban instalarse, puedan hacerlo ahora al encontrar temperaturas más amables. Es urgente, por lo tanto, estudiar mejor las vías de entrada, controlar lo que se pueda en las fronteras, prohibir el comercio y movimiento de especies de las que se conoce su capacidad invasora y evitar el uso de esas especies en nuevas actividades agrarias como los biocombustibles. Aún así no dejaremos de ver aparecer nuevas especies exóticas porque la tendencia es imparable, aunque quizás podamos, si se ponen los medios, reducir en parte su impacto sobre el mundo natural.

\section{Un pacto mundial por los océanos}

La explotación no sostenible de los mares y océanos es uno de los grandes fracasos de la humanidad. A pesar de los múltiples acuerdo concluidos, los valiosos "convenios regionales sobre los mares" (de los que en Europa tenemos unos cuantos afectando al Ártico, al Atlántico, al Báltico, al Mediterráneo, al Mar Negro y al Caspio), 
a pesar de todas las Comisiones Balleneras Internacionales o Comisiones para el Atún Atlántico y a pesar de todos los innumerables acuerdos comerciales de pesca, todos en teoría construidos para asegurar una gestión sostenible del océano y de sus recursos pesqueros y evitar la contaminación por hidrocarburos, plásticos, nitratos, basuras etc., la pura verdad es que los océanos están hechos unos zorros. Los arrecifes coralinos son víctimas del cambio climático y también de métodos insostenibles de pesca. Todos los mares interiores europeos están contaminados en mayor o menor grado y las pesquerías europeas son un pobre recuerdo de lo que fueron un día. El mar parece un territorio sin ley. El respeto de la normativa es la excepción (a pesar de todos los observadores europeos embarcados en los pesqueros y del ingente esfuerzo económico hecho por los contribuyentes europeos para mantener esa industria). Abunda el furtivismo, el poco respeto de los paros biológicos, la captura de alevines, y casi no hay zonas de reserva donde se puedan reproducir las especies a pesar de que eso favorecería a las especies pescadas. Hay demasiada capacidad de pesca, mucha tecnología, y muy poca planificación sostenible. Faltan peces. Siguiendo la lógica de la política y rara vez la de la ciencia se siguen tomando decisiones políticas insostenibles sobre los esfuerzos de pesca. Los mares responden con su progresiva degradación y la tozudez de los hechos. Al agotar los caladeros vamos a buscar otros más distantes, usamos tecnología ultra-sofisticada para levantar peces a cinco kilómetros de profundidad, peces muchos de ellos que han tardado décadas en alcanzar el tamaño con que los sacamos, en una actividad que se asemeja más a la minería de recursos no renovables que a la explotación sostenible. Como en el lema de los juegos olímpicos vamos cada vez mas alto (profundo en este caso), cada vez más rápido, cada vez más lejos. Cada vez dejamos menos a las generaciones siguientes y si alguien (por ejemplo las asociaciones conservacionistas) nos lo reprocha miramos para otro lado.

Esa falta de sensatez debe corregirse. Europa debería poner su influencia y su política en lograr un gran pacto mundial por los océanos que acabe con la actual situación de degradación. Los océanos los conocemos mal, a pesar de que ocupan la mayor parte del planeta. Posiblemente estén en ellos las claves del futuro de la humanidad, su posible despensa, su farmacia, su biblioteca viva más preciada. No pueden seguir siendo sólo basureros, autopistas de barcos y fuentes menguadas de peces. El cambio climático puede afectarlos mucho. Debemos hacer todo lo que esté en nuestra mano para evaluar los bienes económicos inmensos que aportan (no sólo los kilos de pescado, sino los millones de toneladas de gases que absorben su riquísima y mal conocida biodiversidad, su influencia en el clima, su papel en mantener la lluvias, etc.) y ponernos todos de acuerdo en tratarlos de una manera radicalmente distinta. Incluso en los países con gran industria pesquera, como es España, la contribución de la pesca a la riqueza nacional es casi simbólica, con menos del 0,2\% del PIB -y bajando- y no muchas personas en esta actividad. Está claro que hay que ir hacia una disminución de la capacidad de captura, un mayor respeto a los límites ecológicos, y hacia un desarrollo de la acuicultura en que el que mande la sostenibilidad, controlándose también los posibles efectos ambientales. Europa 432 puede liderar ese cambio. 


\section{Controlar el ladrillo, la construcción ubicua de infraestructuras, y otros males: hacia unos nuevos hábitos de consumo sostenibles}

Europa (a la excepción de Rusia) es un continente muy poblado en el que no nos sobra el espacio. Parece claro que una naturaleza conservada no es compatible con densidades muy altas de población. Los recursos ambientales son preciosos para nosotros y no parece sensato malgastarlos en actividades de baja sostenibilidad, como ha sido en los últimos años la desaforada construcción de viviendas secundarias, particularmente en las zonas costeras o la importación de modelos muy poco ecológicos de vivienda, como las viviendas unifamiliares, grandes consumidoras de energía, de espacio y de recursos ecológicos. La ciudad compacta es más sostenible que la dispersa, el bloque de viviendas más ecológico que la urbanización y la densidad urbana es más eficaz energéticamente (en transporte, en calefacción, en consumo de agua) que los adosados, aunque estos nos puedan parecer, a primera vista, más verdes por tener algún que otro árbol y unos metros cuadrados de césped.

Si queremos tener naturaleza y respetar la biodiversidad nuestros modelos de consumo y nuestro modo de vida tendrán que ser sostenibles. Ese es uno de los grandes retos de este siglo que apenas cumple su primera década reflexionando sobre el fracaso de las metas que se había fijado en sostenibilidad del mundo vivo.

Desde la caída del muro de Berlín, episodio con el que terminó simbólicamente un siglo en que Europa fue devastada por guerras terribles y dividida por barreras ideológicas, el desarrollo económico a ultranza ha sido la principal prioridad de los gobiernos. Hemos visto construirse carreteras en todo el Este del continente, sin muchas limitaciones ambientales y el ladrillo ha reinado en el Sur. Todos queremos una Europa próspera y democrática, pero no a costa de cualquier cosa. El desarrollo de los países del Este se ha hecho siguiendo los mismos modelos poco respetuosos del medio ambiente que ya habíamos experimentado en el oeste: infraestructuras enormes, ciudades que dejan poco sitio a la vida, alta velocidad a cualquier precio, ocupación de las costas, abandono del medio rural y gestión intensiva de espacios forestales y agrarios. No hay naturaleza para semejante desarrollo, ni agua para todos los regadíos que queremos inventar, ni montañas para tanta pista de esquí en proyecto, ni kilómetros de costa para tanta ambición de riquezas. Ver pueblos de mil quinientos habitantes haciendo proyectos urbanísticos para alojar veinte o treinta mil personas daría risa si no fuera porque causa espanto: por desgracia tales desaguisados son frecuentes, con el apoyo entusiasta o la indiferencia de partidos de uno u otro signo. Sólo la crisis ha parado tales locuras, que pueden recomenzar cuando pase lo peor. Europa es una pequeña península de Eurasia que tiene sus límites físicos. Es un bello continente cuyo núcleo central -incluyendo Inglaterra- está muy poblado, pero cuyas periferias tienen mucha biodiversidad que conservar, en particular en las penínsulas escandinava, ibérica y balcánica, en las grandes montañas (los Cárpatos, los Alpes, los Pirineos y macizos menores) y en el rosario de islas que circundan el continente (en el Mediterráneo, en el Atlántico, y en el Mar del Norte). Tenemos muchas especies únicas de nuestro continente (en particular en el Sur y en los archipiélagos Atlán- 
ticos -Madeira, Azores, Canarias) y una naturaleza más frágil que otros lugares. No es tarde para conservarla pero hace falta un esfuerzo europeo coordinado y poner freno a la especulación y a los usos no sostenibles del territorio. Las instituciones europeas no han estado ociosas estos últimos años, al contrario, han sabido generar un entusiasmo por la conservación de la biodiversidad a pesar de las reticencias de los gobiernos. La acción política y técnica, incluida la divulgación, de las asociaciones ciudadanas ha sido un elemento fundamental de progreso. Estos grupos se han ido profesionalizando y ganando en seriedad. Un riesgo es que las nuevas generaciones se desinteresen de la conservación del mundo natural, que piensen que es sólo cosa de técnicos o de partidos políticos. Sin una base social sólida será muy difícil que los políticos se lo piensen dos veces antes de autorizar nuevas agresiones al medio ambiente. Esperemos que la grave dimensión de la crisis climática nos haya enseñado algo y vayamos a modelos de sociedad más de acuerdo con el medio en que vivimos. Se puede ignorar la Naturaleza, pero esta es testaruda y nos muestra antes o después nuestros errores. 


\title{
Bonn message High-level working group on the future of global targets for Biodiversity
}

\author{
9-10 march 2009 (Bonn, Germany)
}

\section{INTRODUCTION}

From the 9-10 March 2009 some 50 distinguished participants, at the invitation of the German Federal Ministry for the Environment, Nature Conservation and Nuclear Safety as the current Presidency of the Convention on Biological Diversity $(C B D)$, gathered in Bonn to provide clear and bold direction for future international commitments on biodiversity.

The meeting provided a timely opportunity to discuss the framework for the post 2010 biodiversity target and to prepare the ground for the discussion and action on the global biodiversity agenda. The outcome of the rich discussions at this meeting is a set of suggestions to the UN for further discussions on the future biodiversity targets of the United Nations.

\section{MAIN MESSAGES EMANATING FROM THE MEETING}

\section{A) State and importance of biological diversity}

- Biodiversity and Ecosystems: All life on Earth, including human society, depends upon a variety of services provided by nature, the values of which need wider recognition. Maintaining these services is vital, especially in a world facing massive environmental and economical pressures. This requires that any post 2010 framework ensures the conservation and sustainable use of healthy ecosystems and of the biodiversity upon which their continued functioning depends, as well as the fair and equitable sharing of the benefits arising out of the utilization of genetic resources.

- Biodiversity, Ecosystem Services and Poverty Eradication: The 2010 target provided a valuable framework for biodiversity actions, but missed the 
opportunity to mobilize significant public support and harness adequate political commitment. This could potentially have happened if the critical role of nature and its ecosystem services in supporting human wellbeing had been more clearly incorporated into the 2010 target. For instance, freshwaters, forests, and fisheries are forms of natural capital from which we draw ecosystem services such as clean water, fresh air, carbon storage, food, fibre, and fuel. Nature's services are valuable to the rich and they are irreplaceable and critical for the survival of the poor. Nature is the Treasury of all human beings, especially the Poor. There is no chance for success in poverty eradication and implementing the Millennium Development Goals without well functioning ecosystems at all levels.

- Biodiversity and Climate Change: Healthy ecosystems store carbon but if degraded or destroyed release this as carbon dioxide, one of the principal causes of climate change. Transforming industrial production, energy supply, heating of houses, traffic and transport in a sustainable climate-friendly way is already a tough challenge! Without reducing greenhouse gas emissions originating from the degradation and destruction of ecosystems the challenges of climate change will be hard to achieve. At the same time, climate change is an increasing threat to the maintenance of biodiversity, directly through its potential to accelerate species extinction and indirectly through potentially negative impacts of mitigation measures. Not using synergies between climate change and biodiversity policies would be a double failure -a lose-lose-situation!

- Drivers of Biodiversity loss: Land-use change, eutrophication, the unsustainable use of biological resources, overexploitation of marine ecosystems and invasive alien species continue to be the main drivers of biodiversity loss, but are increasingly exacerbated by climate change. All these drivers of biodiversity loss must be addressed within any post 2010 framework.

- Guidance for a living planet: Any post 2010 target should provide a sense of urgency and be ambitious, simple but meaningful, politically relevant, scientifically credible, inclusive, inspiring, user-friendly and implementable. The new target should consider the key drivers of biodiversity loss and comprise the three objectives of the CBD (conservation, sustainable use, access and benefit sharing)

\section{B) Leadership for biodiversity and ecosystems!}

The global biodiversity target will not be reached by 2010! But there are a number of success stories at sectoral, regional, national and local level. Post 2010 targets should build upon the current 2010 target and the lessons learned from this process.

436 For instance, the target has been useful in introducing biodiversity to the international 
political agenda, raising political and public awareness, building partnerships among institutions and promoting integration of biodiversity in key political processes.

Both medium- and long-term target dates (i.e. 2020 and 2050) should be considered, taking into account existing and other relevant targets, such as those of the Millennium Development Goals. Establishing links between biodiversity and climate change agendas, and other multilateral environmental agreements, should be a priority for any post 2010 framework. In order to ensure measurability, monitoring and assessment of any post 2010 framework, the establishment of baselines, clear milestones and subtargets, and of an agreed set of indicators to measure performance, is important. Key policy makers of different sectors should be involved in the process of defining subtargets and special targets for the local, regional and national levels.

\section{C) Using the crisis for a planet deal!}

The world is facing an economic crisis that may offer opportunities for reforms favouring biodiversity. The valuation of ecosystem services can provide the trigger for change and can serve as a starting point for moving towards a green and sustainable economy. This process can include increased investment in biodiversity as a utility in ecological infrastructure such as bio-diverse, natural carbon sinks and water-purifying wetlands. In this respect, addressing current consumption and production patterns which have a negative impact on the status of biodiversity is also essential. Maintaining biodiversity and ecosystem services also through trade-related agreements could provide a key turning point for any post 2010 biodiversity agenda and for an overall global new deal for our planet. In addition, the ongoing discussions on the post Kyoto climate regime provide a unique opportunity to further advance the post 2010 biodiversity agenda. At the same time the negotiation of an international regime on Access and Benefit Sharing is to be concluded by 2010 as a key element for any post 2010 target. This achievement should be considered in the development of the post 2010 target. Finally, to achieve the necessary paradigm shift it will be essential that the biodiversity community speaks the language of other constituencies, in order to promote synergies among agendas. Building strategic partnerships, including between countries and across different sectors, will assist in enhancing these synergies.

\section{D) Creating broad ownership for the planet deal!}

The post 2010 biodiversity target as part of a global green new deal for our planet has to be adopted at the highest level -the Heads of Government and State- thus providing a mechanism for mainstreaming and policy integration, including horizontal cooperation between ministries. Achieving the target must be incorporated into the mandates of ministries responsible for key sectors -including finance, agriculture, fisheries and forests- with sub-targets specific to those sectors. Any future targets 
should be integrated into relevant national policy instruments such as national biodiversity action plans, national development plans, etc. While Governments should be taking the lead support of stakeholders such as civil society, scientists, indigenous peoples, local communities, and the private sector is vital to obtain broad ownership of all stakeholders in the definition and implementation of any post 2010 target. Defining and implementing future targets and related indicators should be a knowledge driven process informed by science. Instruments that allow for a stronger interface between science and policy, such as the International Platform for Biodiversity and Ecosystem Services (IPBES), should support the implementation of any post 2010 target. "Biodiversity" champions and the mass media are essential to engage present and future generations in order to ensure a broader involvement of society.

\section{THE ROADMAP FOR 2010}

All relevant events in the run up to 2010 should be used to further develop the messages for 2010. The coming 18 months will provide several opportunities to carry forward the discussions around the post 2010 target within and outside biodiversityrelated fora, including events such as those related to the climate change negotiations, the fifth replenishment of the Global Environment Facility and other high-level meetings such as the United Nations General Assembly, G8 and G20 meetings.

2010 as the UN 'International Year for Biodiversity' will be a crucial year for biodiversity in many respects. A sequence of high level conferences allow for an inclusive roadmap leading to a new biodiversity target: in February the global biodiversity community will convene in Trondheim, Norway, to discuss further the elements for a post 2010 target; in February UNEP's Governing Council and Global Ministerial Environment Forum will reconvene; and in May the subsidiary bodies of the CBD (SBSTTA and WGRI) will take stock and advance the post 2010 negotiations, which will feed into the Heads of State Summit that will gather at the United Nations General Assembly (UNGA) in September for a special session devoted to biodiversity; finally, in October the 10th Conference of the Parties to the CBD will meet in Nagoya, Japan. Strong commitments of participants in all these meetings are needed. There is a need to make the difference! 